\title{
Acute Posterior Ischemic Optic Neuropathy Mimicking Posterior Cerebral Artery Stroke Visualized by 3-Tesla MRI
}

\author{
Tilman Menzel Rolf Kern Martin Griebe \\ Michael Hennerici Marc Fatar \\ Department of Neurology, Universitätsmedizin Mannheim, University of \\ Heidelberg, Mannheim, Germany
}

\section{Key Words}

Stroke mimic $\cdot$ Posterior ischemic optic neuropathy $\cdot \mathrm{MRI} \cdot$ Hemianopia

\begin{abstract}
Acute ischemic lesions of the posterior optic nerve and optic tract can produce a variety of visual field defects. A 71-year-old woman presented with acute hemianopia, which led to rtPA thrombolysis for suspected posterior cerebral artery ischemia. 3-Tesla cMRI, however, revealed the cause to be an acute posterior ischemic optic neuropathy. Cases like this may be more common than thought and quite regularly overlooked in clinical practice, especially when there is no high-resolution MRI available. This case strengthens the importance of repeat MR imaging in patients with persistent visual field defects.
\end{abstract}

\section{Introduction}

Acute ischemic lesions of the posterior optic nerve and optic tract can produce a variety of visual field defects.

\section{Case Report}

A 71-year-old female patient was admitted to the emergency room $3.5 \mathrm{~h}$ after onset of left homonymous hemianopia. Apart from a slight right frontal hemicrania, clinical neurological and neuroophthalmological examination was unremarkable. CCT showed only chronic infarctions in the right frontal middle cerebral artery and left posterior cerebral artery territories, and hence the 
patient was treated with rt-PA intravenously (i.v.) according to the results of the ECASS-III-study, unfortunately without improvement of the visual fields.

The following day, the patient reported acute total loss of vision of her right eye; the left eye still had a hemianopia for the left visual field (fig. 1). Although 1.5-Tesla MRI of the brain did not show any acute lesion in DWI or T2* images, the patient was treated with rt-PA i.v. again, this time with half dosage, but again without any effect on the visual loss.

Since the MRI of the brain had failed to show any corresponding lesion(s) for the deficit of the visual field suggesting sequential involvement of the right optic tract and optic nerve including the chiasm, a high-resolution 3-Tesla MRI was performed, which indeed demonstrated an acute DWI and ADC lesion in this area. The nervous tissue also was hyperintense on T2 weighing and showed an enhancement after contrast agent administration (igg. 2).

Ultrasound and biopsy of the right temporal artery demonstrated unspecific thickening of the vessel's intima layer, but no signs for giant cell arteritis. Doppler and duplex sonography as well as MRA established only mild plaques of the large cerebral arteries.

Laboratory studies were inconclusive: systemic vasculitis or neuritis seemed unlikely (ESR 17 mm/1 h, CRP normal; ANA 1:320 (N: 0-1:80), ENA (especially DS-DNS and SSA/SSB) and ANCA were normal; CSF-ACE normal, lues serology negative). However, local atopic inflammation could not be excluded: the screening for anti-cardiolipin and anti-B2-glycoprotein antibodies showed mildly increased titers, while phosphatidylserine antibodies were normal. Rheumatoid factor IgG and IgM were positive.

Total IgE levels were very high with up to $1,895 \mathrm{kU} / \mathrm{l}$ in the serum (N: 0-120) while total IgG and IgM levels were only mildly increased. Mite-specific IgE were very high, especially for Dermatophagoides spp. In contrast, there was no blood eosinophilia in repeat testing and no clinical or radiologic signs indicated pulmonary pathology. The patient's history was unremarkable for asthmatic or other atopic diseases. Lumbar puncture showed no signs of inflammation and was unremarkable otherwise (cell count $1 / \mu \mathrm{l}$, protein $706 \mathrm{mg} / \mathrm{l}$ ).

The patient was treated with methylprednisolone i.v. $1 \mathrm{~g} /$ day for 5 days for possibly associated vascular inflammation, upon which the right frontal headaches quickly remitted. Visual field deficits, however, remained unchanged. A follow-up fundoscopic examination revealed a pale optic disc of the right eye.

\section{Discussion}

This case report illustrates important difficulties in patients suspected to suffer from acute cerebral ischemia of the posterior cerebral circulation, who - different from patients with sensorimotor or speech disturbances due to acute stroke or transient ischemic attacks in the anterior cerebral circulation - are less frequently administered for early thrombolysis in emergency stroke units. While acute hemianopia suggested vascular compromise of the P1 segment of the posterior cerebral artery on early CT and even in the absence of early DWI hyperintensities, sequential total visual loss strongly indicated ischemia in the carotid territory-supplying retina, optic nerve, chiasm and the majority of the optic tract $[1,2]$.

Due to the aforementioned clinical features, MRI and laboratory results, posterior ischemic optic neuropathy (PION) was the most likely diagnosis. Exact epidemiologic data being sparse, PIONs are rare diseases with much lower incidences than anterior ischemic optic neuropathies. Like these, PIONs are divided etiologically into arteritic and nonarteritic types [1]. 
A possible differential diagnosis was arteritis limited to intracranial vessels, e.g. an intracranial granulomatous arteritis or a primary angiitis of the central nervous system; the good clinical condition and follow-up of our patient as well as normal CRP, the near-normal ESR as well as the unspecific results of the biopsy of the temporal artery excluded this diagnosis [3, 4]. However, the very high total and mite-specific IgE titers might have suggested a vascular involvement in an atopic inflammation of central nervous tissue, which has only come to attention in recent years but is still not well defined as a clinical entity so far [5].

\section{Conclusion}

Acute PION is a rare cause of visual field defects and might have been overlooked more often than reported, in particular if careful and selective MR-based neuroimaging of the optic nerve, chiasm and tract is not performed. In the acute phase only a few hours after onset, the diagnosis is even more difficult: this is probably why such a subtle lesion closely mimicking acute stroke in the territory of a posterior cerebral artery, has not been reported so far. Failure of collateral blood supply within the extensive arterial network from the carotid artery is similarly rare - thus, only blockage of small penetrating arteries branching off the intracranial siphon of the carotid artery could account for this highly specific ischemic pattern.

This case strengthens the importance of repeat MR imaging in patients with persistent visual field defects despite normal acute MR imaging - furthermore, high resolution imaging of the visual system should be performed in all cases of ophthalmologically undetermined visual field defects.

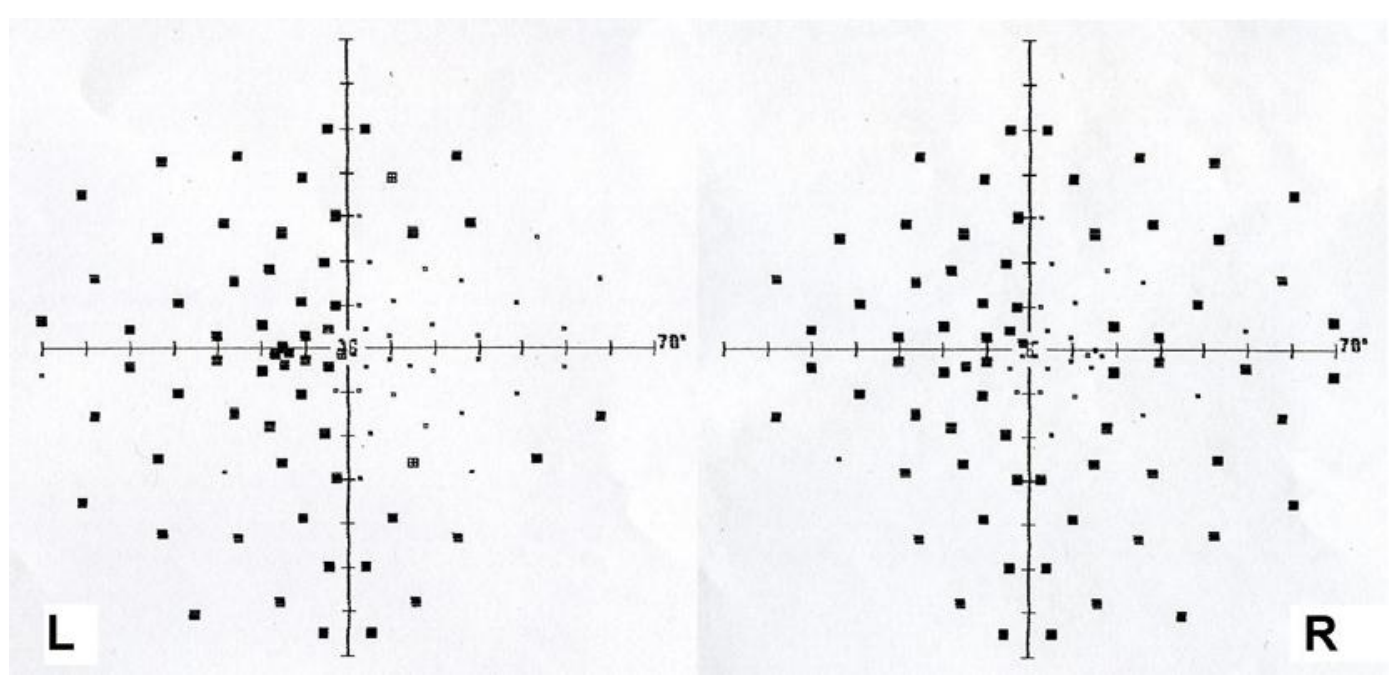

Fig. 1. Computer-aided perimetry of the patient's visual fields demonstrating the combined homonymous hemianopia to the left of both eyes and amaurosis of the right eye. 


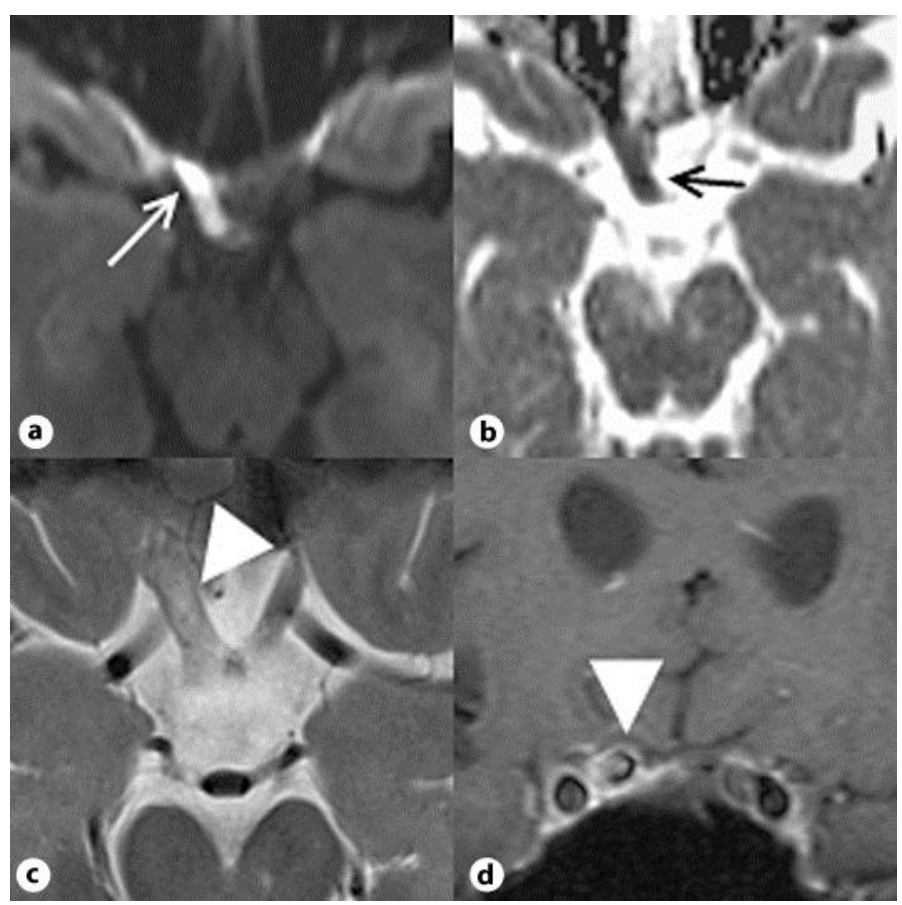

Fig. 2. a, b Notable diffusion restriction with corresponding ADC lesion of the posterior right optic nerve and the right optic chiasm (arrows). c, d T2-weighted hyperintensity and enhancement of contrast agent in T1-weighted imaging of the posterior right optic nerve and the right optic chiasm (arrowheads). a-c Transverse plane, $\mathbf{d}$ coronal plane.

\section{References}

1 Hayreh SS: Ischemic optic neuropathy. Prog Retin Eye Res 2009;28:34-62.

-2 van Overbeeke J, Sekhar L: Microanatomy of the blood supply to the optic nerve. Orbit 2003;22:81-88.

-3 Hajj-Ali RA, Calabrese LH: Central nervous system vasculitis. Curr Opin Rheumatol 2009;21:10-18.

4 Birnbaum J, Hellmann DB: Primary angiitis of the central nervous system. Arch Neurol 2009;66:704709.

5 Isobe N, Kira J, Kawamura N, Ishizu T, Arimura K, Kawano Y: Neural damage associated with atopic diathesis: a nationwide survey in Japan. Neurology 2009;73:790-797. 